Int. J. Dev. Biol. 51: 131-137 (2007)

doi: $10.1387 / \mathrm{ijdb} .062226 \mathrm{ok}$

Original Article

\title{
The importance of the posterior midline region for axis initiation at early stages of the avian embryo
}

\author{
ODED KHANER* \\ Department of Health Sciences, Hadassah Academic College, Jerusalem, Israel
}

\begin{abstract}
The avian blastoderm acts during its early stages of development as an integrative system programmed to form a single embryonic axis. Here, I report the results of a variety of transplantation experiments of the midline region at stages X-XII, which were carried out to study their relevance for axis initiation. The results of the experimental series discussed herein emphasizes the importance of the posterior midline region (including the marginal zone and Koller's sickle) for axis initiation. This ability resides mainly at stage $X$ in the posterior side of a narrow midline region, while at stages XI-XII it is exhibited at the region which is located more anterior and lateral to the posterior midline region. This posterior midline region has developmental abilities which allow it to initiate a single embryonic axis and at the same time to prevent other regions that also have such abilities to do so. Therefore, in normal development only one embryonic axis develops in the avian blastoderm. It is proposed that the cells which are important to initiate the avian embryonic axis are concentrated mainly at the region of the posterior midline region. These cells may have organizer properties which determine the initiation site of the axis in the avian embryo.
\end{abstract}

KEY WORDS: avian embryo, axis initiation, posterior midline region, transplantation $\neq$ of midline region

\section{Introduction}

Axis formation in the avian embryo is a complex integrative and sequential process. The axis is a highly organized structure with distinct polarity that develops from the unpolarized blastodisk. The fertilized avian egg at its earlier stages I-VI shows a distinct radial symmetry (Eyal-Giladi and Kochav, 1976). However, during the next stages of interuterine development, (VII-X, EG\&K) the posterior-anterior axis is first determined by the formation of the polarized area pellucida. After the egg is laid, this polarity is superimposed upon the blastoderm during the next stages of development by sequential polarized morphogenetic processes. First, the development of Koller's sickle and the hypoblast, stages (XI-XVI, EG\&K) and later on, the development of primitive-streak, stages 2-4 (Hamburger and Hamilton, 1951). By the primitive streak stages, the avian embryo becomes polarized along the anterior-posterior, the dorso-ventral and the left-right axes (Khaner, 1993).

In normal development, only one primitive streak is formed in the avian blastoderm. However, if the chick blastoderm at its early stages of development can be cut into as many as eight pieshaped fragments and separated, each fragment can spontaneously give rise to a miniature embryonic axis (Spratt and Haas,
1960). These experiments confirm the finding that any region of the pre-primitive streak stage embryo has the ability to form an embryonic axis. The fact that anterior half or even lateral parts of intact embryos never do so during normal development suggests the existence of endogenous inhibitory factors, from whose influence the anterior or lateral parts are released upon cutting.

Recently, experiments were done to investigate the signaling pathways involved in primitive streak induction (Skromne and Stern, 2001; Skromne and Stern, 2002). Other experiments followed the signaling pathways that ensure that only a single embryonic axis forms in the blastoderm. It was found that $\mathrm{Vg} 1$ induces an inhibitor that travels across the embryo. This inhibitor acts early in the cascade of events downstream of $\mathrm{Vg} 1$. It was suggested that three sequential inhibitory steps ensure that a single axis develops in normal development of the embryo (Bertocchini et al., 2004).

In other research an attempt was made to learn whether the two halves of the avian blastoderm, when shifted along the midline and then reunited in staggered fashion, act as one or two separate embryonic fields. When a reunion of the right and the left halves was achieved so that the posterior end of one half was adjoining the posterior area pellucida region of the other half, a single embryonic axis developed. When, in contrast, the shift was

*Address correspondence to: Dr. Oded Khaner. Department of Health Sciences, Hadassah Academic College, P.O.B 1114, Jerusalem, 91010 Israel.
Fax: +972-2-6250619. e-mail: odedk@hadassah-col.ac.il

$0214-6282 / 2007 / \$ 30.00$

(c) UBC Press

Printed in Spain

www.intjdevbiol.com 

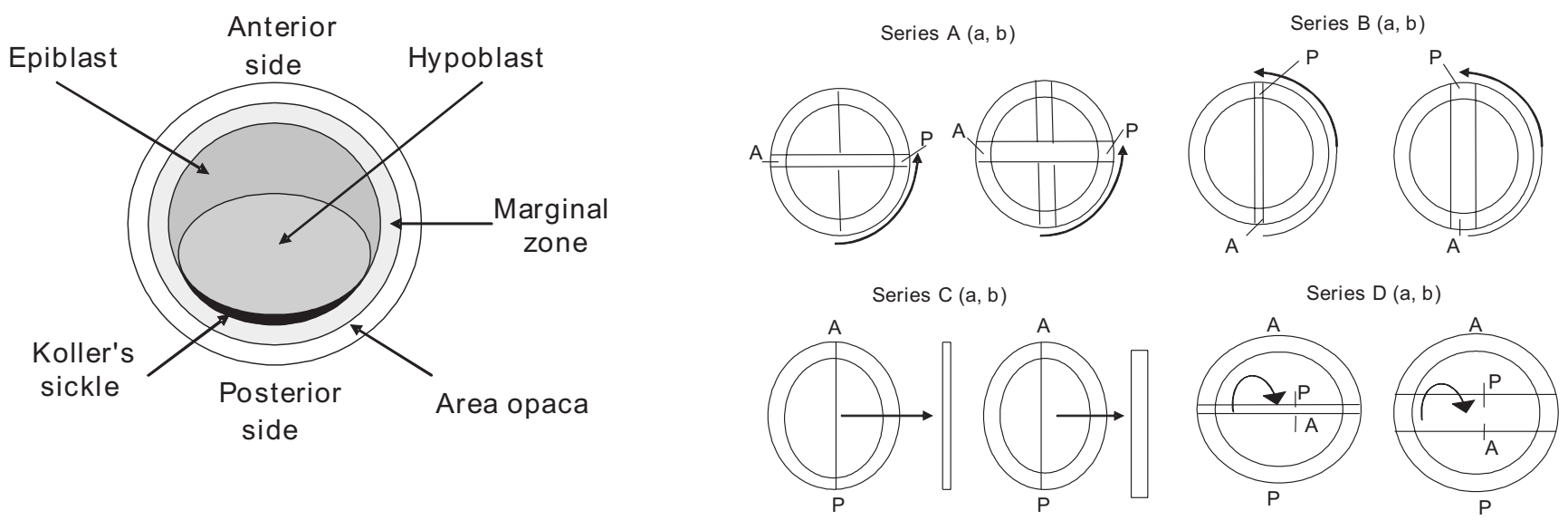

Fig. 1 (Left) Scheme of a stage XII avian blastoderm before operation viewed from its ventral side (anterior side up and posterior side down).

Fig. 2 (Right). Schemes of experimental procedures (Series A-D). In all of the experimental procedures, the narrow strip of midline cells included an area of about 1/9 from blastoderm diameter and the wide strip of midline cells included an area of about 2/9 from the blastoderm diameter. (Series Aa) A narrow strip from both sides of the midline was dissected vertically. A horizontal cut was made and the strip of midline cells was then transplanted horizontally into the gap between the two halves. (Series Ab) A wide strip from both sides of the midline was dissected vertically. Horizontal cuts were made and a wide strip was cut into two pieces. The midline strip was then reciprocally exchanged with both pieces of the horizontal strip. (Series B) A narrow (Ba) or a wide (Bb) strip from both sides of the midline was dissected vertically and reverse transplanted. (Series C) A narrow (Ca) or a wide (Cb) strip from both sides of the midline was dissected vertically and discarded. (Series $\mathbf{D}) \mathrm{A}$ narrow (Da) or a wide (Db) horizontal strip was dissected vertically and reciprocally transplanted. (In this particular case (Db), the wide strip of midline cells included an area of about 3/9 from blastoderm diameter). Abbreviations: $A$, anterior; $P$, posterior.

larger so that the posterior end was fused to the central area pellucida of the other half, two separated embryonic axes developed (Khaner, 1996). These experiments demonstrated the importance of the midline region of the avian blastoderm in the process of axis initiation.

The aim of this research was to study the developmental potentials of the midline region of the avian blastoderm (stages $X$ XII). Experiments were designed to isolate the midline region, to transplant it into ectopic positions and to follow its ability to initiate the embryonic axis.

\section{Results}

The following experimental manipulations were conducted in this study (Figs. 2,3,4 and Table 1).

\section{Horizontal transplantation of the midline region (Series A: a and $b$ )}

During normal development, primitive streak initiation starts at the posterior side of the blastoderm. To study the developmental abilities of the midline region to initiate an ectopic embryonic axis, different types of manipulations were done.

(a) A narrow strip from both sides of the midline was dissected vertically and moved aside before transplantation. A horizontal cut was made separating the operated blastoderm into posterior half and anterior half. The strip of midline cells was then transplanted horizontally (left or right) into the gap between to two halves (Figs. 2, 3A). The results obtained demonstrated that at stage $X$ most of the operated blastoderms developed an embryonic axis from the original posterior side of the horizontal transplanted strip (86\%). After about 36-48 hours, the embryonic axis appeared normally and included brain, notochord and somites
(Fig. 4A). However, at stages XI-XII, most of the operated blastoderms developed two embryonic axes. One axis developed from the original posterior side of the horizontal transplanted midline strip, while the other axis developed from the original posterior side of the blastoderm (XI - 85\%, XII - 70\%). Neither streak completed its development into a mature embryonic axis (Fig. 4B).

(b) A wide strip from both sides of the midline was dissected vertically and moved aside before transplantation. Horizontal cuts were made and a wide strip (the same size as the midline strip) was moved aside and cut into two pieces. The midline strip was then reciprocally exchanged (left or right) with both pieces of the horizontal strip (Figs. 2, 3B). The results obtained in this series of experiments demonstrated that at all stage (X-XII) an embryonic axis developed from the original posterior side of the horizontal transplanted strip (X - 67\%, XI - 70\%, XII - 60\%).

\section{Reversal transplantation of the midline region (Series B: a and $b)$}

The results obtained from experimental series A demonstrated the developmental abilities of the posterior midline region to initiate the embryonic axis, when transplanted $90^{\circ}$ (left or right) close to the original posterior side of the blastoderm. What will however be the consequences if the posterior midline region will be transplanted far $\left(180^{\circ}\right)$ from the original posterior side of the blastoderm?

To examine that question other types of manipulations were done.

(a) A narrow strip from both sides of the midline was dissected vertically and reverse transplanted (Fig. 2). The results obtained demonstrated that at stage $X$ most of the operated blastoderms developed an embryonic axis from the original posterior side of 
the reversal transplanted strip (75\%). However, at stages XI-XII, in most of the operated blastoderms three embryonic axes developed (XI $71 \%$, XII - 57\%). One axis developed from the original posterior side of the reverse transplanted strip. The other two axes developed from both sides of the original posterior region of the blastoderm. In most of the cases, these axes appeared normally. Their development was however limited to a mature primitive streak (Fig. 4C).

(b) A wide strip from both sides of the midline was dissected vertically and reverse transplanted (Figs. 2, 3C). The results obtained in this series of experiments demonstrated that at all stages (XXII) a mature primitive streak and later on a young embryonic axis developed from the original posterior side of the reversal transplanted strip (100\%) (Fig. 4D).

\section{Dissecting and discarding of the midline re- gion (Series $C$ : $a$ and $b$ )}

The results obtained from experimental series $A$ and $B$ demonstrated the developmental abilities of the posterior midline region to initiate the embryonic axis. What will however be the consequences if a narrow or wide midline region will be discarded?

To examine that question other types of manipulations had been done.

(a) A narrow strip from both sides of the midline was dissected vertically and discarded (Figs. 2, 3D). The results obtained demonstrated that in all of operated blastoderms of all stages (X$\mathrm{XII}$ ) a mature primitive streak and later on a young embryonic axis
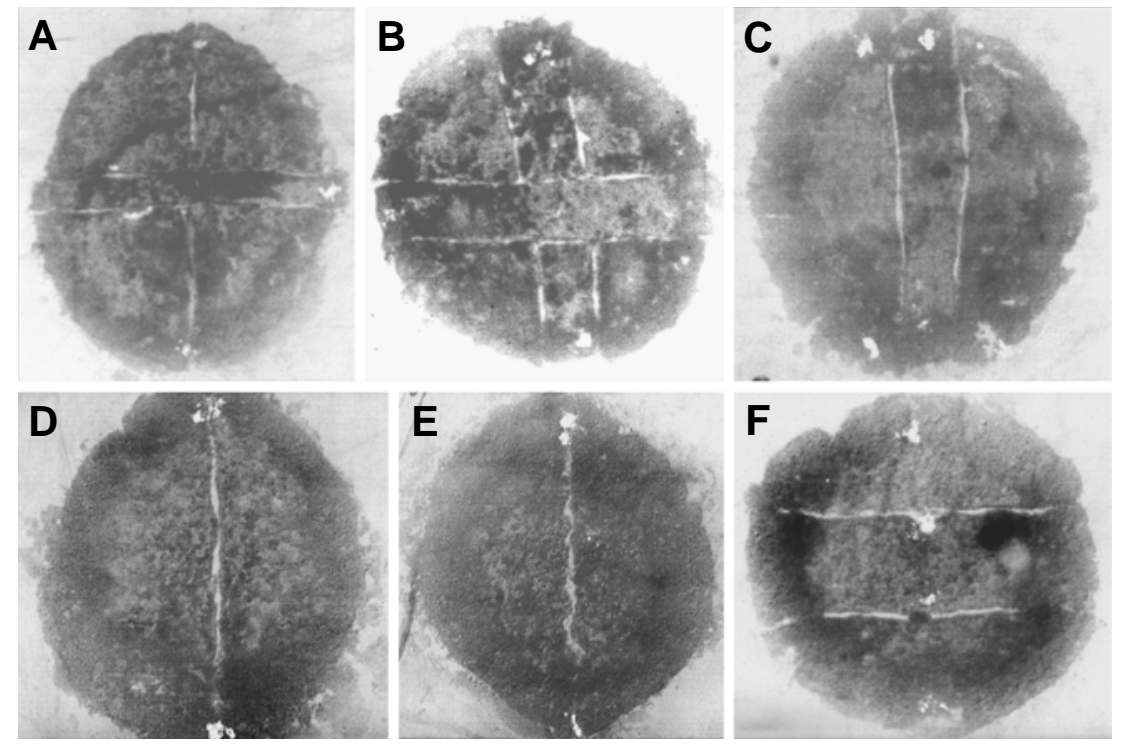

Fig. 3. Operated blastoderms viewed from their ventral side (anterior side up and posterior side down). (A) A stage XI blastoderm at the end of the specific series $A a$ operation. (B) A stage XI blastoderm at the end of the specific series Ab operation. (C) A stage XII blastoderm at the end of the specific series Bb operation. (D) A stage XI blastoderm at the end of the specific series Ca operation. (E) A stage XI blastoderm at the end of the specific series Cb operation. (F) A stage X blastoderm at the end of the specific series Db operation. Magnification: (X36) throughout.

which included brain, notochord and somites developed from the original posterior side of the blastoderm (100\%) (Fig. 4E).

(b) A wide strip from both sides of the midline was dissected vertically and discarded (Figs. 2, 3E). When such a manipulation was done, just unorganized mesoderm with no polarity developed in all blastoderms from stages (X-XII).

Fig. 4. Operated blastoderms after 24-48 $\mathrm{h}$ of incubation viewed from their ventral side (anterior side up and posterior side down). (A) A stage Xblastoderm at the end of 48 h of incubation series Aa operation. An embryonic axis developed from the original posterior side of the horizontal transplanted strip. The embryonic axis appeared normally and included B-brain, $N$-notochord and $S$-somites and HN-Hensen's node. (B) A stage XI blastoderm at the end of 24 h of incubation series Ab operation. Two embryonic primitive streaks developed. One streak developed from the original posterior side of the horizontal transplanted midline strip, while the other streak developed from the original posterior side of the blastoderm. (C) $A$ stage XI blastoderm at the end of $24 \mathrm{~h}$ of incubation series Ba operation. Three primitive streaks developed, one streak developed from the original posterior side of the reverse transplanted strip. The other two streaks developed from both sides of the original posterior region of the blastoderm. PS, primitive streak. (D) A stage X blastoderm at the end of $28 \mathrm{~h}$ of incubation series Bb operation. A mature PSprimitive streak developed from the original posterior side of the reversal transplanted midline strip. PS, primitive streak: HN, Hensen's node. (E) A stage XI blastoderm at
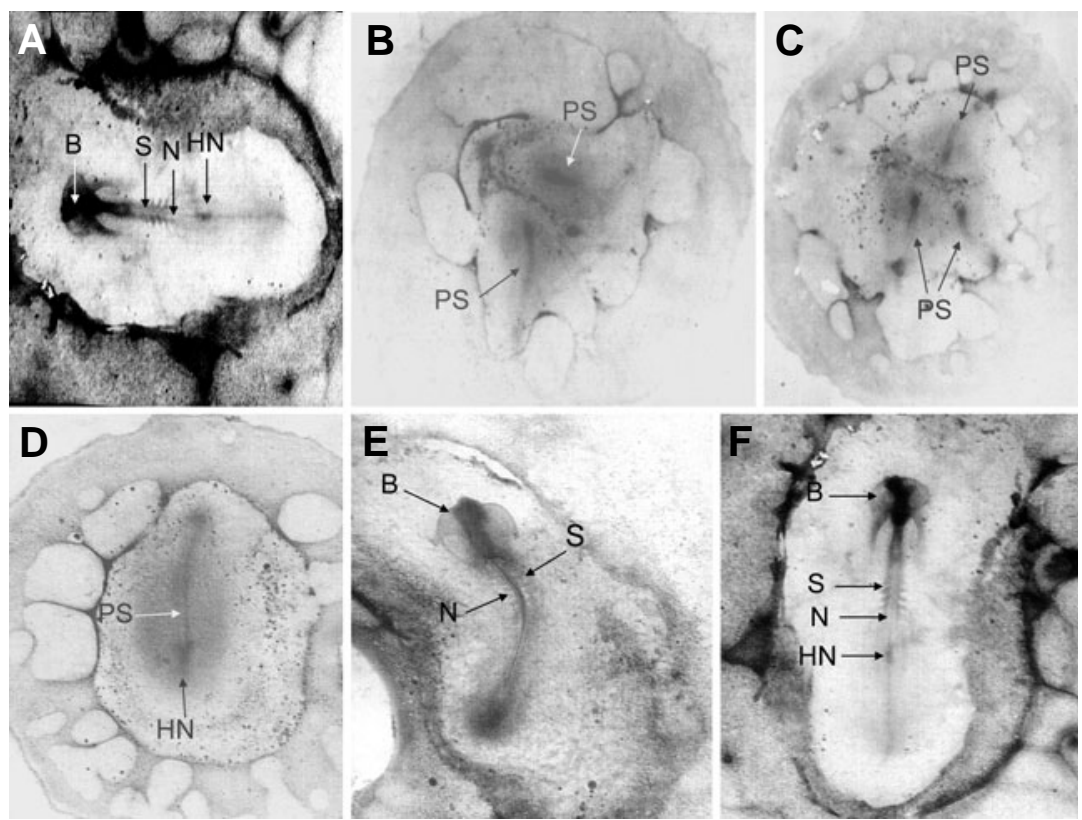

the end of $36 h$ of incubation series Ca operation. Embryonic axis which included B-brain, N-notochord and S-somites developed from the original posterior side of the blastoderm. (F) A stage XI blastoderm at the end of $36 \mathrm{~h}$ of incubation series Da operation. Embryonic axis which included Bbrain, N-notochord and S-somites developed from the original posterior side. HN, Hensen's node. Magnification: (X36) throughout. 


\section{Reciprocal transplantation of a horizontal strip of cells (Se- ries $D$ : $a$ and $b$ )}

The different types of manipulations reported in series A, B and $C$ included always the midline region. However, in series $D$, an attempt was made to split this region, particularly at its posterior side and to follow the consequences of such a split on axis initiation.

(a) A narrow horizontal strip was dissected vertically and reciprocal transplanted (left to right and right to left). In such manipulation, the midline region at its posterior side was left intact (Fig. 2). The results obtained demonstrated that in all operated blastoderms of all stages (X-XII), a mature primitive streak and later on a young embryonic axis (which included brain, notochord and somites) developed from the original posterior side of the blastoderm (X - 80\%, XI-67\%, XII - 57\%) (Fig. 4F).

(b) A wide horizontal strip (in this particular series an area of about $3 / 9$ from blastoderm diameter) was dissected vertically and reciprocal transplanted (left to right and right to left) (Figs. 2, 3F). In such a manipulation, the midline region at its posterior side was split. The results obtained from stage $X$ demonstrated that most of the operated blastoderms developed a mature primitive streak and later on a young embryonic axis developed from the original posterior side of the blastoderm (73\%). However, at stages XI-XII, most of the operated blastoderms developed two embryonic axes (XI - 63\%, XII - 71\%). One axis developed from the original posterior side of the blastoderm while the other axis developed from the posterior side of the transplanted strip. Neither streak completed its development into a mature embryonic axis.

Control: Twenty five blastoderms from stages X- XII (EG\&K) were operated and in all of them different types of dissections (series A-D) were made and left intact in their place. In most of these control blastoderms (85\%), a mature normal embryonic axis developed from the original posterior side of the blastoderm.

\section{Discussion}

At early stages of development, an embryonic axis with distinct anterior-posterior polarity is established in the avian blastoderm. This polarity starts to develop at the uterine stages. At later stages, this polarity is superimposed upon the blastoderm in formation of a polarized axis (Khaner, 1993).

In normal development, only one primitive streak is formed in the avian blastoderm. However, different types of experiments demonstrated that the avian embryo is an extremely regulative system. Any fragment of the avian embryo right up to the beginning of primitive streak formation can form a complete axis when isolated. None of these regions other than the posterior part do so in intact embryo. This suggested that there must be a control mechanism which contributes to the formation of a polarized axis and also inhibits the tendencies of other regions to initiate an axis. These reports support the notion that a region responsible for axis initiation exists at the posterior side of the area pellucida (Spratt and Haas, 1960; Spratt, 1963; Khaner and Eyal-Giladi, 1986; Khaner and Eyal-Giladi, 1989; Khaner, 1993; Khaner, 1998, Bertocchini et al., 2004). Moreover, it was found that the midline region of the avian blastoderm has a crucial role in the process of axis formation (Khaner, 1996).

The experiments reported here followed directly the developmental potentials of the midline region of the avian blastoderm
TABLE 1

\section{RESULTS OF ALL OPERATED BLASTODERM (SERIES A-D, STAGES X-XII)}

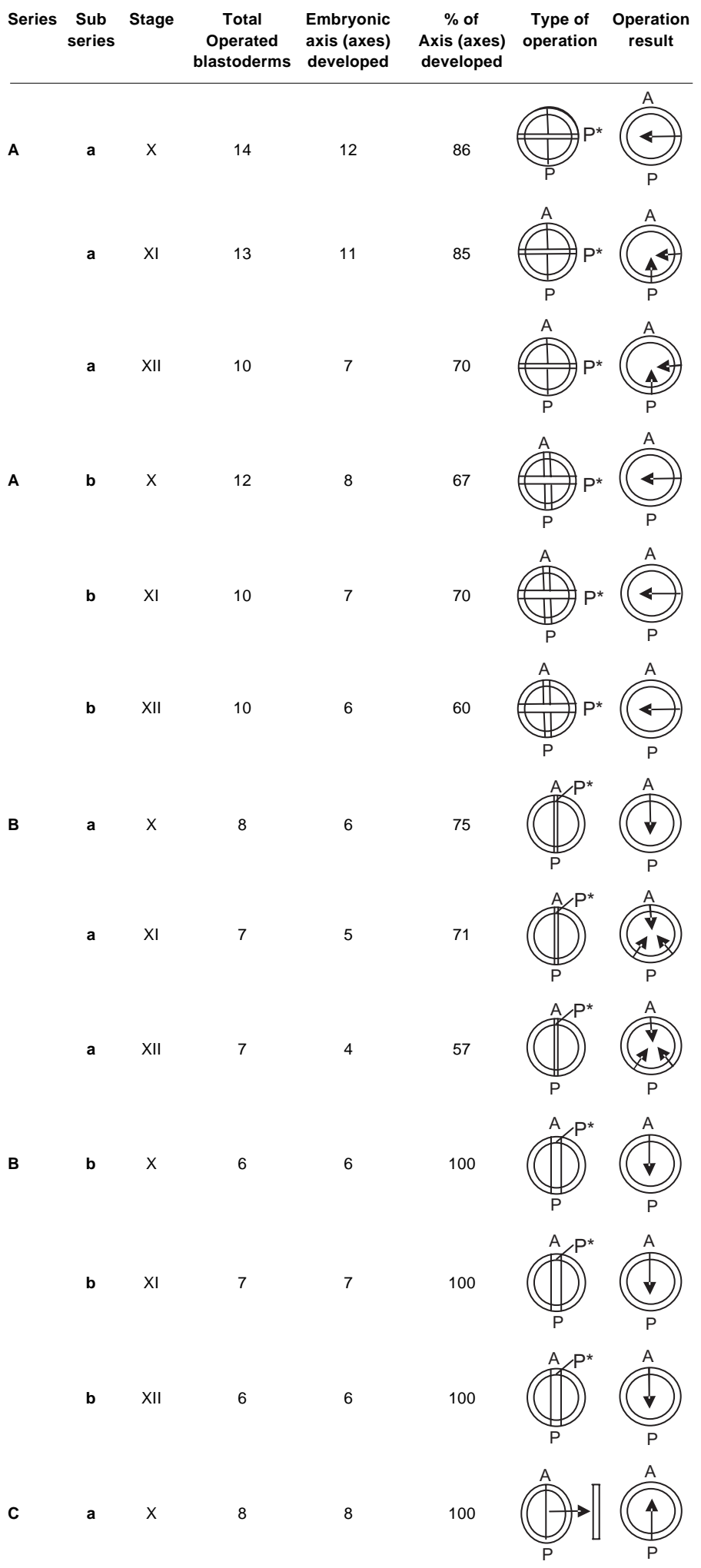




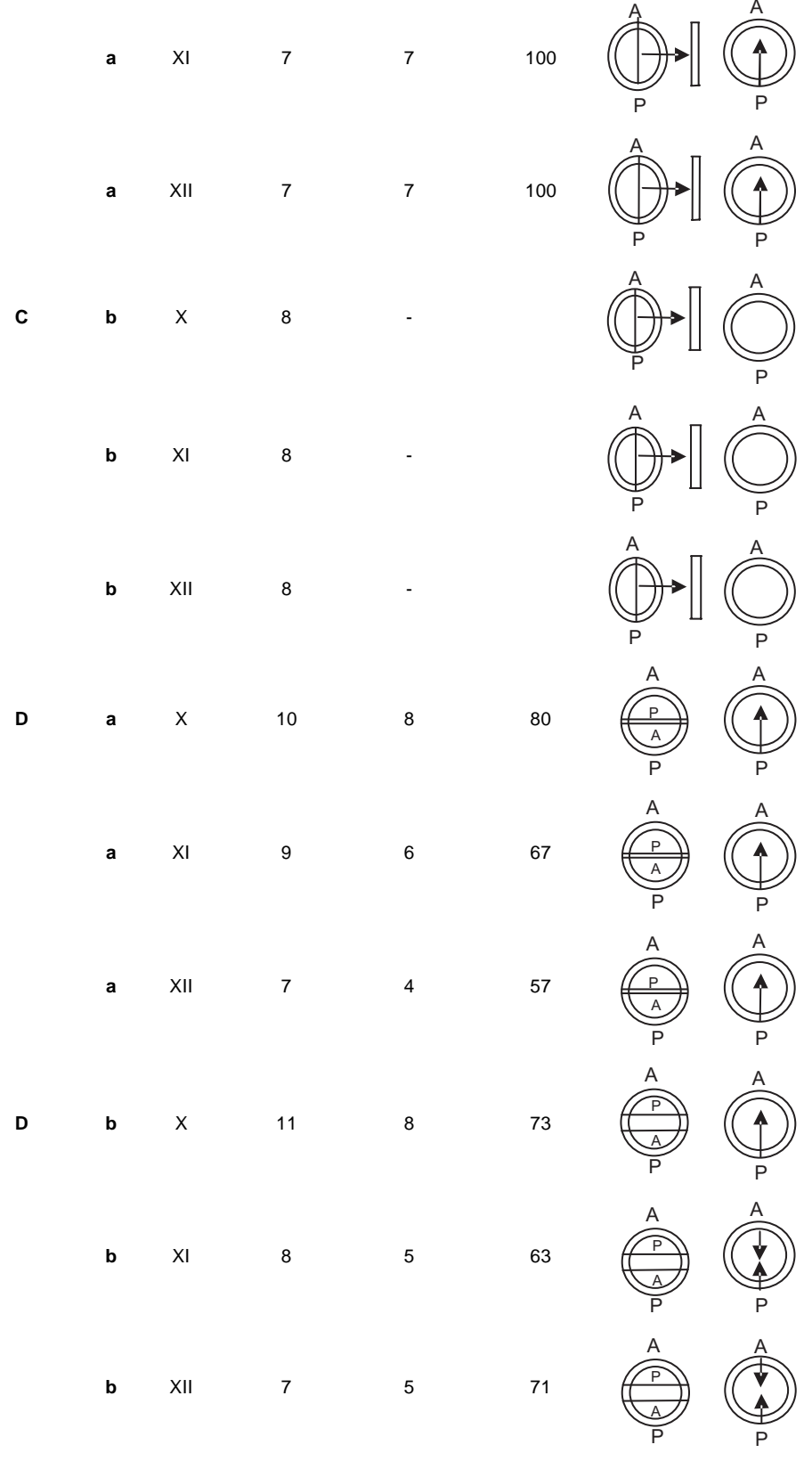

A - Anterior, P - Posterior, PS - $\rightarrow$

(stages X-XII). All series of experiments concentrate mainly on the developmental potential to initiate a single embryonic axis of the midline area and especially its posterior region. In series $A$ and $B$ narrow and wide strips of midline cells were transplanted in horizontal $(A)$ and in reverse $(B)$ positions, respectively. Whenever this experiment was done with a stage $X$ blastoderm, the embryonic axis was initiated from a narrow posterior midline region of the transplant. Therefore, this result demonstrates clearly that this particular posterior midline region has the developmental potential to initiate the development of a single embryonic axis at stage $X$. Experiments of series $C$ examined the inhibition effect of this particular region. When a narrow strip of midline cells is discarded (a), an embryonic axis still developed, but in this case from both adjacent posterior-lateral sides of the midline. Namely, posterior-lateral sides of the posterior midline have the developmental potential to initiate an embryonic axis. However, during normal development this region is inhibited by the posterior midline region which was discarded in this experiment.

The importance of this region to axis initiation comes also from series $\mathrm{D}(\mathrm{a} ; \mathrm{b})$. As a result of experimental manipulation at stage $X$, this particular posterior midline region was left intact at its original position in both experiments $(a$ and $b$ ). The results demonstrate that the axis was initiated in both types of experiments from this particular posterior midline region.

The results obtained in this study from manipulated blastoderms at stages XI-XII, demonstrate that the developmental potential to initiate embryonic axis still resides in the narrow posterior midline region. However, in contrast to stage $X$, this developmental potential transfers also to more lateral and anterior regions of the posterior side. In series $A$ and $B$ the midline strip was transplanted horizontal or in reverse positions. When a wide strip of midline cells was transplanted ectopically $A(b)$ and $B(b)$, only one embryonic axis developed from the posterior side of the transplant. However when a narrow strip of midline cells was transplanted ectopically $A(a)$ and $B(a)$, two and even three embryonic axes developed respectively in the same blastoderm. One can predict that at stages XI-XII (in contrast to stage X), the developmental potential to initiate the embryonic axis transferred also to more lateral and anterior regions of the posterior side. Indeed, in both cases although the midline region was transferred ectopically, lateral and anterior parts from both sides of the posterior midline region also possess developmental potentialities. These regions which stayed at their original location could initiate an embryonic axis, in addition to the axis which was initiated ectopically from the original posterior midline region. In series $\mathrm{A}(\mathrm{a})$ only one axis developed from this region because both sides of the midline were put together tightly. However at series $\mathrm{B}(\mathrm{a})$ there was a gap of anterior midline cells between this region. In this case each separate side act independently and therefore two axes developed. Similar results were obtained when glass beads were put as an intact gap between both sides of this region (Khaner and Eyal-Giladi, 1989).

In series $\mathrm{D}(\mathrm{b})$ at stages XI-XII, a split was made at this particular region. Upon reciprocal transplantation of a wide horizontal strip, one part of this region stayed at its original place, while, the other part of this region was transferred into anterior position. In this case, although a split was made in this particular region, each part had the developmental potential to initiate a unique axis.

The results of the experimental manipulation reported here (series $\mathrm{Aa}, \mathrm{Ba}$ and $\mathrm{Db}$ ), show that two or even three distinct primitive streaks were developed in the same blastoderm, however, neither streak completed its development into a mature embryonic axis. These results are consistent with previous reports in which two distinct streaks in the same blastoderm frequently do not complete their development into a mature embryonic axis. (Khaner and Eyal-Giladi, 1986; Khaner and Eyal-Giladi, 1989; Khaner, 1998). The explanations for such a phenomenon could be the fact that in these types of manipulations, the developmental potential to form a mature embryonic axis was split into two or three separate regions. Each of these separate regions 
could still promote the formation of a primitive streak, but this streak did not have sufficient ability to continue its development into a mature embryonic axis.

What is the meaning of inhibition if two or even three axes could develop in the same blastoderm? A recent publication indicated that $\mathrm{Vg} 1$ induces an inhibitor that travels across the embryo $(3 \mathrm{~mm}$ distance) in less than 6 hours (Bertocchini et al., 2004). Although such inhibitors exist, they could not prevent the initiation of more than one embryonic axis (series $\mathrm{Aa}, \mathrm{Ba}$ and $\mathrm{Db}$ ). As it looks from the experiments reported here, the location of the regions which could initiate the axes were relatively far from the source of inhibitors and therefore it could not prevent axes formation. In addition, it is known that several other manipulations (such as marginal zone grafts, or certain types of hypoblast rotation), as well as in some series in this report, also generate ectopic axes. In these cases, this is not considered as conflicting with the proposed inhibitors, but rather, they help to explain where and when the various inhibitors might act (Bertocchini et al., 2004).

The results obtained from all series of experiments in this study indicate the role of the posterior midline region of the avian blastoderm for axis initiation. In recent publications a controversy arose over the importance of different parts of the posterior region of the avian blastoderm (posterior marginal zone and Koller's sickle) for axis initiation (Callebaut and Van Nueten, 1994; Khaner, 1998; Bertocchini et al., 2004). Previous studies identified the posterior marginal zone of the avian embryo at stage $X$, as the most crucial region for axis initiation (Khaner and Eyal-Giladi, 1986; Khaner and Eyal-Giladi, 1989). A more recent study identified the posterior marginal zone of the avian embryo, as the functional equivalent of the Nieuwkoop center of amphibians (Bachvarova et al., 1998). In this report, the posterior region of the marginal zone and Koller's sickle stayed intact. Dissections were made only from both sides of the midline which included both the posterior marginal zone and Koller's sickle (A, B and C), or anterior to that region (D).

This study stresses the importance of the posterior midline region to initiate the embryonic axis at stage X-XII. In the chick embryo, goosecoid, (which in amphibia is expressed in the prospective head mesoderm portion of Spemann's organizer), is expressed in the same site during stages X-XII (Izpisua-Belmonte etal., 1993). It might be that the posterior midline region may have organizer properties which determine the initiation site of an embryonic axis. Moreover, $c V g 1$ which is a powerful inducer of organized axial tissue amphibia, is also expressed in the same site of the posterior midline region, at stages X-XIII (Seleiro et al., 1996). The pattern of expression of the genes, goosecoid and cVg1, seems to converge with the particular posterior midline region, which was found in this work to express axis initiation ability.

What is the overall role of the midline region for axis initiation and formation? Recent publications indicate that the midline in its middle region expresses Nodal, the main signaling molecule involved in induction of the mesoderm (Bertocchini and Stern, 2002). Moreover, the posterior midline region of the chick blastoderm was found to be a region of cell death that is essential for separation of the left and the right sides of the embryo (Kelly et al., 2002). Other studies showed oriented cell division along this midline, which was proposed to drive elongation of the primitive streak (Zahavi et al., 1998; Wei and Mikawa, 2002). Could these results indicate the mechanisms explaining some of the results seen in this report? Thoughts on this issue are necessary and valuable and might be put under investigation in the future. As we know, different factors such as: Vg1, Wnt8C, Nodal, Chordin, BMP and FGF appear to act during the process of axis development. Therefore, different types of experiments might be done to establish the precise nature of the contribution of each of these signaling pathways to axis formation. Such experiments for example will require combining misexpression of one of the signaling molecules with inhibition of each of the other pathways in turn.

In conclusion, the experiments reported here emphasis the importance of the posterior midline region (including the marginal zone and Koller's sickle) for axis initiation at stage $X$. This ability resides mainly at stage $X$ in the posterior side of a narrow midline region. At stages $\mathrm{XI}-\mathrm{XII}$ the ability to initiate the axis is exhibited at the region which is located more anterior and lateral to the posterior midline region. This particular region of the posterior midline region has developmental abilities which allow it to initiate a single embryonic axis and on the same time to prevent other regions that also have such abilities to do so. Therefore, in normal development only one embryonic axis develops in the avian blastoderm.

\section{Materials and Methods}

Fertile white Leghorn chick eggs were used as freshly laid eggs, stage $X,(E G \& K)$. Other eggs were incubated for approximately 4-8 hours at $37.5^{\circ} \mathrm{C}$, until the blastoderms reached stages XI-XII (EG\&K). Eggs were rinsed with $70 \%$ alcohol and opened into a bowl filled with Ringer's solution. A large round piece of vitelline membrane, with the blastoderm in its center, was dissected with scissors, freed carefully from the yolk and transferred (lower side up) into a Petri dish containing Ringer's solution. A glass ring, $1.5 \mathrm{~cm}$ in diameter, was placed on the vitelline membrane with the blastoderm at its center. The blastoderm was cleaned of all remaining adhering yolk and the exact stage of the blastoderm was identified. The prospective anterior-posterior polarity was determined on the basis of Koller's sickle and the growing hypoblast (Fig. 1). The edges of the vitelline membrane were then stretched and folded over the glass ring and the glass ring with the blastoderm was transferred into a sterilized Petri dish containing thick egg albumin (New, 1955).

The blastoderms were operated according to published procedures (Khaner, 1998). Briefly, dissections were made with a fine tungsten needle or hair loop. The posterior side of the blastoderm was always marked with carbon particles (black) on the region of the area opaca, while the anterior (opposite) side was marked with carmine particles (red).

In all of the experimental manipulations, the narrow strip of midline cells included an area of about $1 / 9$ from blastoderm diameter and the wide strip of midline cells included an area of about 2/9 from blastoderm diameter. The transplanted midline region included also the regions of the marginal zone and the area opaca to allow better regeneration of the operated blastoderms. The operated blastoderms were incubated in a humid environment for $24-48$ hours at $37.5^{\circ} \mathrm{C}$. During the incubation period, the embryos were monitored, photographed and analyzed with reference to stages of the normal table of chick development $(\mathrm{H} \& \mathrm{H})$. The orientation of the developed embryonic axis was determined by observation of carbon particles (in the posterior side of the blastoderm and in posterior position of the graft) and of carmine particles (in the anterior side of the blastoderm and in the anterior position of the graft).

All of the manipulated blastoderms from all stages continued to develop after the operation. In most of the blastoderms, a primitive streak and later on a mature embryonic axis developed. Other operated blastoderms developed. However, a primitive streak could not be observed. In 
these blastoderms, just unorganized mesoderm with no polarity could be detected.

\section{References}

BACHVAROVA, R.F., SKROMER, I. and STERN, C.D. (1998). Induction of primitive streak and Hensen's node by the posterior marginal zone in the early chick embryo. Development 125: 3521-3534.

BERTOCCHINI, F. and STERN, C.D. (2002). The hypoblast of the chick embryo position the primitive streak by antagonizing nodal signaling. Dev. Cel/3: 735744.

BERTOCCHINI, F., SKROMER, I., WOLPERT, L. and STERN, C.D. (2004). Determination of embryonic polarity in a regulative system: evidence for endogenous inhibitors acting sequentially during primitive streak formation in the chick embryo. Development 131: 3381-3390

CALLEBAUT, M. and VAN NUETEN, E. (1994). Rauber's (Koller's) sickle: The early gastrulation orgenizer of the avian blastoderm. Eur.J Morphol. 32: 35-48.

EYAL-GILADI, H. and KOCHAV, S. (1976). From cleavage to primitive streak formation: a complementary normal table and a new look at the first stages of the development of the chick. I. General morphology. Dev. Biol. 49: 321-337.

HAMBURGER, V. and HAMILTON, H. L. (1951). Stages in the development of the chick embryo. J. Morphol. 88: 49-92.

IZPISUA-BELMONTE, J. C., DE ROBERTIS, E. M., STOREY, K. G. and STERN, C. D. (1993). The homeobox goosecoid and the origin of organizer cells in the early chick blastoderm. Cel/67: 89-104.

KELLY, K.A., WEI, Y and MIKAWA, T. (2002). Cell death along the embryo midline regulates left-right sidedness. Dev. Dynamics 224: 238-244.

KHANER, O. and EYAL-GILADI, H. (1986). The embryo forming potency of the posterior marginal zone in stage X through XII of the chick. Dev. Biol. 115: 275281.

KHANER, O. and EYAL-GILADI, H. (1989). The chick's marginal zone and primitive streak formation. I. Coordinative effect of induction and inhibition. Dev. Biol. 134: 206-214.

KHANER, O. (1993). Axis determination in the avian embryo. In «Curr. Top. Dev.
Biol.» (R. Pedersen, Ed.), Vol. 28, pp. 155-180. Academic Press, Inc

KHANER, O. (1996). Axis formation in half blastoderm of the chick: stage of separation and relative position of fused halves influence axis. Roux's Arch. Dev. Biol. 205: 364-370.

KHANER, O. (1998). The Ability to Initiate an Axis in the Avian Blastula is Concentrated Mainly at a Posterior Site. Dev. Biol. 194, 257-266.

NEW, D. A. T. (1955). A new technique for the cultivation of the chick embryo in vitro. J. Embryol. Exp. Morphol. 3: 326-331.

SELEIRO, E. A. P., CONNOLLY, D. J. and COOKE, J. (1996). Early developmental expression and experimental axis determination by the chicken Vg1 gene. Curr. Biol. 6: 1476-1486.

SKROMNE, I. and STERN, C.D. (2001). Interactions between Wht and Vg1 signaling pathways initiate primitive streak formation in the chick embryo. Development 128: 2915-2927.

SKROMNE, I. and STERN, C.D. (2002). A hierarchy of gene expression accompanying induction of the primitive streak by $\mathrm{Vg} 1$ in the chick embryo. Mech. Dev. 114: $115-118$.

SPRATT, N. T. and HAAS, H. (1960). Integrative mechanisms in development of early chick blastoderm. I. Regulative potentiality of separated parts. J. Exp. Zool. 145: 97-137.

SPRATT, N.T. (1963). Role of substratum supracellular continuity and differential growth in morphogenetic cell movements. Dev. Biol. 7: 51-72.

WEI, Y. and MIKAWA, T. (2002). Formation of the avian primitive streak for spatially restricted blastoderm: evidence for polarized cell division in the elongating streak. Development 127: 87-96.

ZAHAVI, N., RIECH, V. and KHANER, O. (1998). High proliferation rate characterizes the site of axis formation in the avian bastula-stage embryo. Int. J Dev Biol. 42: 95-98.

Received: 18th July 2006 Reviewed by Referees: 10th August 2006 Modified by Authors and Accepted for Publication: 26th September 2006 Published Online: 2nd February 2007

\section{Other Int. J. Dev. Biol. articles of interest}

See our Special Issue on The Spemann Mangold Organizeredited by E. De Robertis and J. Aréchaga at: http://www.ijdb.ehu.es/web/contents.php?vol=45\&issue=1

The expression and alternative splicing of alpha-neurexins during Xenopus development. Zhihong Zeng, Colin R. Sharpe, J. Paul Simons and Dariusz C. Górecki. Int. J. Dev. Biol. (2006) 50: 39-46.

Systematic screening for genes specifically expressed in the anterior neuroectoderm during early Xenopus development Noriyuki Takahashi, Naoko Tochimoto, Shin-Ya Ohmori, Hiroshi Mamada, Mari ltoh, Masako Inamori, Jun Shinga, ShinIchi Osada and Masanori Taira. Int. J. Dev. Biol. (2005) 49: 939-951.

Patterning a multi-headed mutant in Hydractinia: enhancement of head formation and its phenotypic normalization. Werner A Müller, Regina Teo and Frank Möhrlen. Int. J. Dev. Biol. (2004) 48: 9-15.

The Spemann-Mangold organizer: the control of fate specification and morphogenetic rearrangements during gastrulation in Xenopus. T Bouwmeester. Int. J. Dev. Biol. (2001) 45: 251-258.

Early neurogenesis in Amniote vertebrates. NM Le Douarin. Int. J. Dev. Biol. (2001) 45: 373-378.

Otx2 and HNF3beta genetically interact in anterior patterning. O Jin, K Harpal, $S \angle$ Ang and J Rossant. Int. J. Dev. Biol. (2001) 45: 357-365. 